\title{
Temporal and Spatial Interactions between the Carotid Sinus and Vagally Mediated Baroreflexes in Dogs
}

\author{
Hiroshi Hosomi, Hiroyasu Yoshida, Yasuhiro Nishida, \\ Hironobu Morita, Mitsuhiro Kawada, Nobuhisa Uemura, \\ and Syozo Koyama \\ Department of Physiology, Kagawa Medical School, \\ Kida-gun, Kagawa, 761-07 Japan
}

\begin{abstract}
We investigated the temporal and spatial interactions between the carotid sinus and aortic arch baroreflex control of arterial pressure in 25 dogs anesthetized with pentobarbital sodium. The carotid sinus baroreceptor region was vascularly isolated to control the intracarotid sinus pressure. A hemorrhage catheter was inserted into the aortic arch. The systemic arterial pressure change after quick mild hemorrhage $(2 \mathrm{ml} / \mathrm{kg}$ body weight within 1-2s) was monitored. The open-loop gain of the vagally mediated baroreflex system was estimated from the mean arterial pressure response to the hemorrhage. Three protocols were employed to analyze the interactions. In the first protocol, we determined the effect of different levels of intracarotid sinus pressure on the open-loop gain of the vagally mediated baroreflex system. There was no significant effect. In the second protocol, the open-loop gain of the carotid sinus baroreflex system was determined after vagotomy. In the third protocol, the vagally mediated baroreflex system was activated by the hemorrhage without (spatial interaction) or with (temporal interaction) a delay after changing the intracarotid sinus pressure. The spatial interaction was facilitatory. A temporal interaction was found between the carotid sinus and vagally mediated baroreflex systems, when the delay was less than $30 \mathrm{~s}$.
\end{abstract}

Key words : temporal interaction, spatial interaction, carotid sinus baroreflex, vagally mediated baroreflex, open-loop gain.

The baroreceptors located in the carotid sinus, aortic arch and cardiopulmonary regions send detailed information on the arterial and venous pressures to the medulla via the carotid sinus, aortic and vagus nerves. The central nervous system receives and integrates these signals, and sends efferent signals to the cardiovascular system which influence the arterial blood pressure. How these signals

Received for publication July 26, 1986 
are integrated has been the subject of numerous reports (NINOMIYA and IRISAWA, 1969; ANGELL-JAMES and DALY, 1970; KöTTER et al., 1970; KATONA and TAN, 1975; Hosomi and SAgAwa, 1979; Kendrick et al., 1979; Hosomi et al., 1981, 1986; IsHIKAWA and SAGAWA, 1983; BRUNNER et al., 1984). Although many studies examined the spatial interaction between/among these baroreflex systems, there is much disparity in the literature. Such conflicting results may reflect differences in experimental conditions, i.e., electrical or pressure stimulus, changes in nerve activity or pressure, conscious or anesthetized, and kind of species.

On the other hand, a few studies (KöTTER et al., 1970; KATONA and TAN, 1975; KENDRICK et al., 1979) have been concerned with the subject of temporal interaction. In these studies, a delay between electrical stimulation of carotid sinus and aortic nerves was changed. KATONA and TAN (1975) and KENDRICK et al. (1979) changed the delay below one second and found no significant temporal interaction between both baroreflexes. However, KöTTER et al. (1970) found that the pressure reduction caused by electrical stimulation of the carotid sinus nerve is enhanced if preceded by a $30 \mathrm{~s}$ stimulation of the aortic nerve. These results suggest that there may be a facilitatory temporal interaction between the baroreflex systems if a delay between the baroreflexes is more than one second.

In the present investigation, we employed pressure stimuli as an input to the carotid sinus and vagally mediated baroreflex systems, and measured the reflexogenic pressure changes as an output of the systems in anesthetized dogs. To induce the carotid sinus baroreflex without eliciting any carotid chemoreflex effect, we isolated the carotid sinuses and decreased their static pressure. The vagally mediated baroreflex was elicited by hypotension induced by hemorrhage. We elicited the carotid sinus and vagally mediated baroreflexes simultaneously or separately, and compared the combined reflex with the sum of the separate reflexes. We also elicited the vagally mediated baroreflex with a relatively large delay after elicitation of the carotid sinus baroreflex, and examined the effect of the preceding reflex by the carotid sinus baroreflex system on the vagally mediated baroreflex.

\section{METHODS}

Animals and procedures. Results were obtained from a total of 25 mongrel dogs of either sex, weighing $10.9 \pm 0.4$ (S.E.) $\mathrm{kg}$. The dogs were anesthetized by intravenous injection of pentobarbital sodium $(35 \mathrm{mg} / \mathrm{kg}$ body weight) and heparinized with 500 units $/ \mathrm{kg}$ body weight of sodium heparin. The dogs were then intubated and allowed to breathe room air spontaneously. A rectal probe was inserted to monitor body temperature, which was maintained at about $38^{\circ} \mathrm{C}$. The systemic arterial pressure was measured via a catheter inserted into the right omocervical artery. The mean systemic arterial pressure (MAP) was derived from the systemic arterial pressure with a low-pass filter with a $2 \mathrm{~s}$ time constant and recorded on a strip chart. The right and left carotid sinuses were vascularly isolated from the rest of the circulatory system. The occipital, internal, and external carotid 
arteries as well as any small branches originating from the carotid bifurcation were completely ligated. The intracarotid sinus pressure (ICSP) was controlled, monitored, and recorded on the strip chart. A large bore catheter for hemorrhage was advanced into the aortic arch via the left subclavian artery. The other end of the catheter was connected to a piston-syringe system. The piston shaft was connected to a handle with a joint. Displacement of the handle caused a displacement of the piston and hemorrhage. The displacement of the handle was imparted by pulling the handle by hand. The hemorrhage volume was accurately reproduced by setting a stopper on the shaft of the handle prior to hemorrhage. The displacement of the handle was transformed into an electric signal by a sliding resistance attached to the handle shaft and recorded on the strip chart.

Experimental protocols and data analysis. Three protocols were employed to investigate the temporal and spatial interactions between the carotid sinus and vagally mediated baroreflex systems. In the first protocol with $10 \mathrm{dogs}$, we determined the effects of the intracarotid sinus pressure on the barostatic response of the vagally mediated baroreflex system to hemorrhage. We set the intracarotid sinus pressure at $25,75,125$, or $175 \mathrm{mmHg}$ in a semirandom order. Ten min later and after the systemic arterial pressure had become stable, $2 \mathrm{ml}$ of blood per $\mathrm{kg}$ body weight were quickly withdrawn into the syringe within $1-2 \mathrm{~s}$ from the aorta (quick mild hemorrhage). This quick mild hemorrhage caused a rapid fall in the systemic arterial pressure and then activated the rapidly acting arterial pressure control system, i.e., the vagally mediated baroreflex system in this protocol, which restored the systemic arterial pressure to the control level and minimized the systemic arterial pressure fall. We could estimate the open-loop gain $\left(G_{\mathrm{V}}\right)$ of the vagally mediated baroreflex system from the systemic arterial pressure change as described previously (Hosomi et al., 1983). Briefly, we sampled the control mean arterial pressure (CMAP) just before the hemorrhage and then the immediate fall in mean arterial pressure $\left(\triangle \mathrm{AP}_{\mathrm{I}}\right)$ from the control mean arterial pressure at completion of the hemorrhage. We measured the steady-state fall in mean arterial pressure $\left(\triangle \mathrm{AP}_{\mathrm{S}}\right)$ from the control mean arterial pressure. In practice, we sampled $\triangle \mathrm{AP}_{\mathrm{S}}$ every $6 \mathrm{~s}$ from 1 to $2 \mathrm{~min}$ after the hemorrhage and averaged the results. The static overall open-loop gain $(G)$ of the negative feedback control system can be defined as follows (Milhorn, 1966; Hosomi and Yokoyama, 1981):

$$
G=\Delta \mathrm{AP}_{\mathrm{I}} / \Delta \mathrm{AP}_{\mathrm{S}}-1 \text {. }
$$

The intracarotid sinus pressure was changed several times and the quick mild hemorrhage experiment was performed once at each change of intracarotid sinus pressure. The blood drawn into the syringe was retransfused into the aorta $2 \mathrm{~min}$ after the hemorrhage. The measurements were pooled at each intracarotid sinus pressure level in individual dogs. The average values were again pooled for the 10 dogs. The statistical significance of the difference in changes in the measurements caused by the quick mild hemorrhage at different intracarotid sinus pressures was first evaluated by two-way analysis of variance. When the resultant values were 
found to be statistically significant, the difference between any pair of measurements at two intracarotid sinus pressures was tested by the paired $t$-test.

In the second protocol with $7 \mathrm{dogs}$, we vascularly isolated the carotid sinus baroreceptors and sectioned the vagus nerves at the cricocartilage level. About 30 $40 \mathrm{~min}$ later and after the systemic arterial pressure had become stable, the intracarotid sinus pressure was changed in steps of $20 \mathrm{mmHg}$ from 180 to $40 \mathrm{mmHg}$ (carotid sinus open-loop experiment). One and a half minutes later, we measured the increase $(\triangle \mathrm{AP})$ in the mean systemic arterial pressure. The open-loop gain of the carotid sinus baroreflex system $\left(G_{\mathrm{CS}}\right)$ was estimated as follows:

$$
G_{\mathrm{CS}}=\Delta \mathrm{AP} / 20 \text {. }
$$

This carotid sinus open-loop experiment was repeated a few times. The measurements were pooled within individual dogs and pooled again for the 7 dogs.

In the third protocol with $8 \mathrm{dogs}$, we examined the temporal interaction between the carotid sinus and vagally mediated baroreflex systems. After the intracarotid sinus pressure and the systemic arterial pressure had established equilibrium, we decreased the intracarotid sinus pressure by about the same amount as the immediate fall in mean systemic arterial pressure which had been determined previously in the quick mild hemorrhage experiment in the same dog. Without or with a delay $(5,15,30,60$, or $120 \mathrm{~s})$ after the change in intracarotid sinus pressure, the quick mild hemorrhage experiment was performed. This hemorrhage experiment was repeated at least twice for each delay. The immediate and steady-state falls in mean arterial pressure were measured and the open-loop gain was determined as described above. The measurements were pooled within the experiment with the same delay in individual dogs, and pooled again for the 8 dogs. The statistical significance of the effects of the delay on the responsiveness of the vagally mediated baroreflex system to the quick mild hemorrhage was first evaluated by two-way analysis of variance. When the resultant values were found to be statistically significant, the difference between any pair of measurements at two delays was tested by the paired $t$-test. The results are presented as the mean \pm S.E.

\section{RESULTS}

Protocol 1: Effects of intracarotid sinus pressure level on the open-loop gain of the vagally mediated baroreflex system

An example of this experimental protocol is shown in Fig. 1. The control mean systemic arterial pressure decreased significantly with increasing intracarotid sinus pressure (Table 1$)$. The immediate fall $\left(\Delta \mathrm{AP}_{\mathrm{I}}\right)$ in mean systemic arterial pressure after the hemorrhage ranged from -17.3 to $-19.4 \mathrm{mmHg}$ and was not affected by the level of intracarotid sinus pressure. The immediate fall in the mean systemic arterial pressure was gradually restored and the mean systemic arterial pressure reached a steady-state in 1 to $2 \mathrm{~min}$. The steady-state fall $\left(\Delta \mathrm{AP}_{\mathrm{S}}\right)$ in mean systemic arterial pressure ranged from -7.6 to $-8.9 \mathrm{mmHg}$ and was not affected by the level 


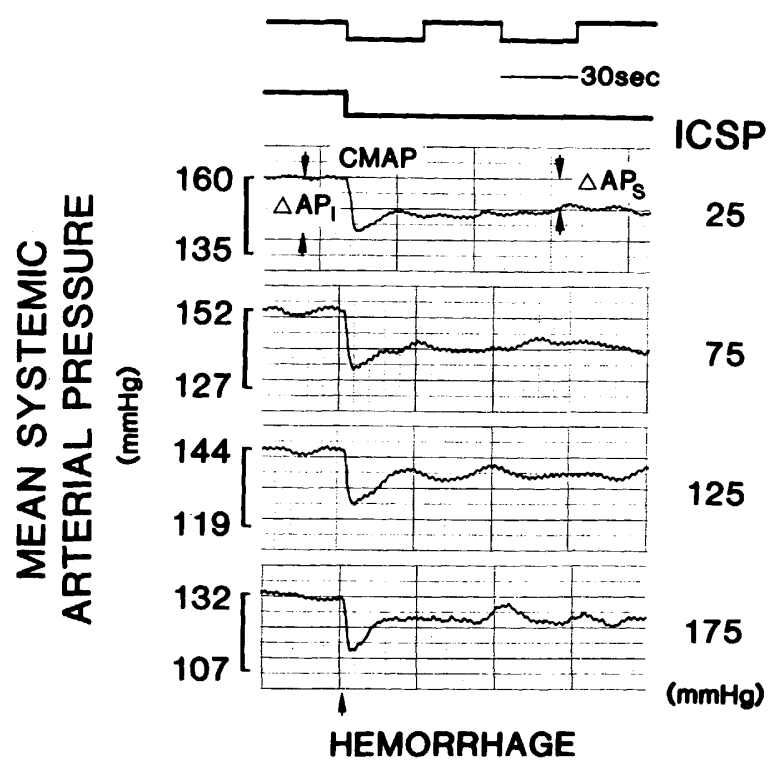

Fig. 1. Typical mean systemic arterial pressure responses of the vagally mediated baroreflex system to quick mild hemorrhage at different intracarotid sinus pressure levels. Upper trace, time; second trace, change in bled volume; third to sixth traces, mean systemic arterial pressure responses to hemorrhage at intracarotid sinus pressures (ICSP) of $25,75,125$, and $175 \mathrm{mmHg}$, respectively. CMAP, control mean arterial pressure; $\triangle \mathrm{AP}_{\mathrm{I}}$, immediate fall in mean systemic arterial pressure; $\triangle \mathrm{AP}_{\mathrm{S}}$, steady-state fall in mean systemic arterial pressure over 1 to $2 \mathrm{~min}$ after hemorrhage (see text).

of intracarotid sinus pressure. Thus, the mean systemic arterial pressure response to quick mild hemorrhage did not change depending on the different levels of intracarotid sinus pressure. These results suggested that the level of intracarotid sinus pressure could not affect the open-loop gain estimated from $\triangle \mathrm{AP}_{\mathrm{I}}$ and $\triangle \mathrm{AP}_{\mathrm{S}}$. Since the open-loop gain was calculated for each hemorrhage experiment and then pooled, the average gain value could not be estimated from the pooled $\Delta \mathrm{AP}_{\mathrm{I}}$ and $\triangle \mathrm{AP}_{\mathrm{S}}$.

\section{Protocol 2: Open-loop gain of the carotid sinus baroreflex system}

The mean open-loop gain of the carotid sinus baroreflex system was calculated for the different levels of intracarotid sinus pressure by dividing the change in systemic arterial pressure by $20 \mathrm{mmHg}$. The data obtained are presented in Table 2 . The region where the maximum gain was observed lies between 120 and $140 \mathrm{mmHg}$. 
Table 1. Effects of intracarotid sinus pressure level on control mean arterial pressure (CMAP), immediate fall in mean arterial pressure $\left(\triangle \mathrm{AP}_{\mathrm{I}}\right)$, steady-state fall in mean arterial pressure $\left(\triangle \mathrm{AP}_{\mathrm{s}}\right)$, and openloop gain of the vagally mediated baroreflex system $\left(G_{\mathrm{v}}\right)$.

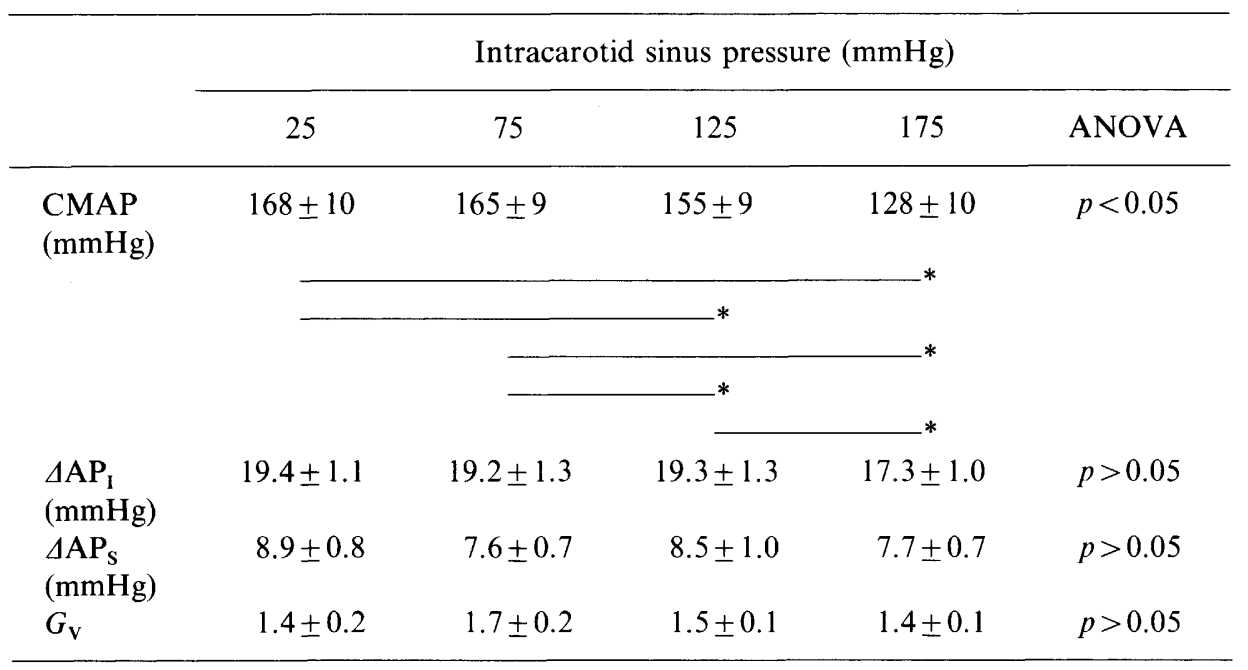

Values are mean \pm S.E. $(n=10) .{ }^{*} p<0.05$, paired $t$-test.

Protocol 3: Temporal interaction between the carotid sinus and vagally mediated baroreflexes

An example of this experimental protocol is shown in Fig. 2. The intracarotid sinus pressure was decreased stepwise by about $20 \mathrm{mmHg}$ from the equilibrium level. In response, the mean systemic arterial pressure increased and became stable within less than $5 \mathrm{~s}$. The change in intracarotid sinus pressure was followed by hemorrhage with different delays. The hemorrhage caused a rapid arterial pressure fall and then activated the vagally mediated baroreflex system which restored the arterial pressure towards the control. The immediate fall in mean systemic arterial pressure changed slightly but significantly with increasing delay (Fig. 3). The steadystate fall in mean systemic arterial pressure increased significantly with increasing delay (Fig. 3). The open-loop gain of the rapidly acting arterial pressure control system was $6.7( \pm 0.9)$, when both the carotid sinus and vagally mediated baroreflex systems were unloaded simultaneously. The open-loop gain of the vagally mediated baroreflex system was $1.5( \pm 0.1)$ (Protocol 1) and that of the carotid sinus baroreflex system was $1.9( \pm 0.3)$ (Protocol 2). The open-loop gain (6.7) of simultaneous stimulation was thus larger than the sum $(3.4=1.5+1.9)$ of the gains of the separate stimulations. This finding suggests that there is a facilitatory interaction between the two reflexes, when both reflexes are elicited simultaneously. The gain decreased progressively with increasing delay, and became $1.2( \pm 0.2)$ when the delay was $120 \mathrm{~s}$ (Fig. 4). This gain value was not significantly different 
Table 2. Open-loop gain of the carotid sinus baroreflex system $\left(G_{\mathrm{CS}}\right)$ at different intracarotid sinus pressures.

\begin{tabular}{cccccccc}
\hline & \multicolumn{7}{c}{ Intracarotid sinus pressure (mmHg) } \\
\cline { 2 - 8 } & $180-160$ & $160-140$ & $140-120$ & $120-100$ & $100-80$ & $80-60$ & $60-40$ \\
\hline$G_{\mathrm{CS}}$ & $0.4 \pm 0.4$ & $1.3 \pm 1.0$ & $1.9 \pm 0.7$ & $1.5 \pm 0.7$ & $0.9 \pm 0.6$ & $0.2 \pm 0.4$ & $0.1 \pm 0.1$ \\
\hline
\end{tabular}

Values are mean \pm S.E. $(n=7)$.

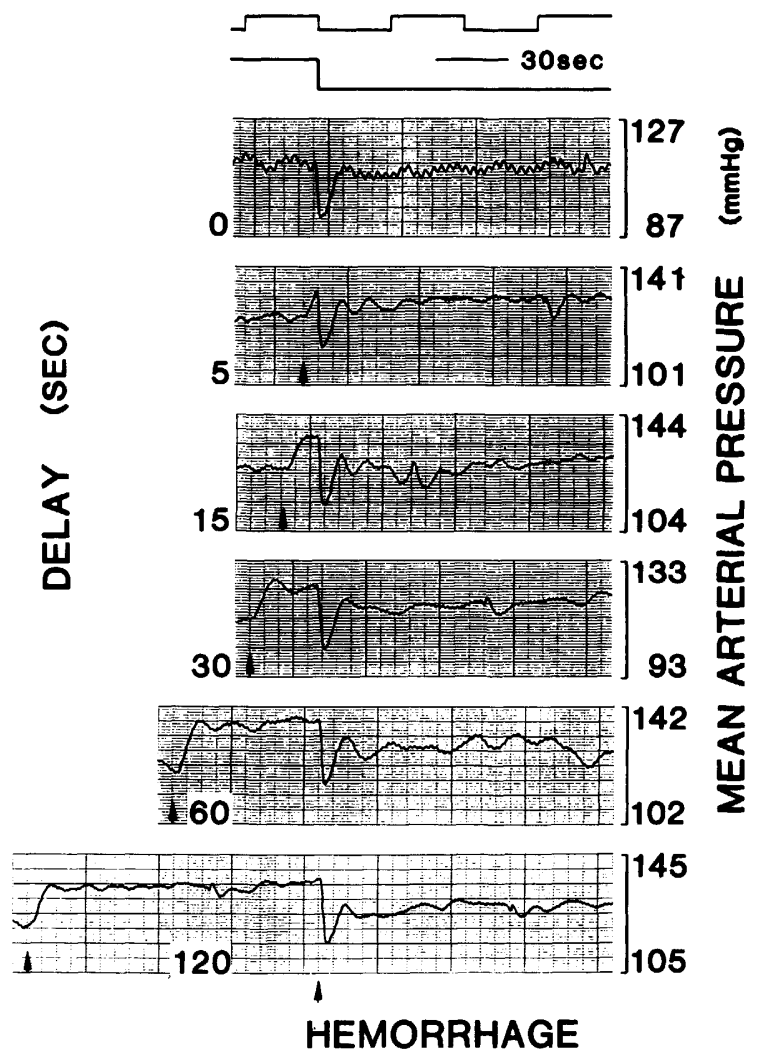

Fig. 2. Typical mean systemic arterial pressure responses to decrease in intracarotid sinus pressure by $20 \mathrm{mmHg}$ and quick mild hemorrhage. The decrease in intracarotid sinus pressure preceded the quick mild hemorrhage by different delays (DELAY).

from the open-loop gain $(1.5 \pm 0.1)$ of the vagally mediated baroreflex system. Therefore, this gain value represented that of the vagally mediated baroreflex system and was not affected by the preceding (120 s) activation of the carotid sinus 


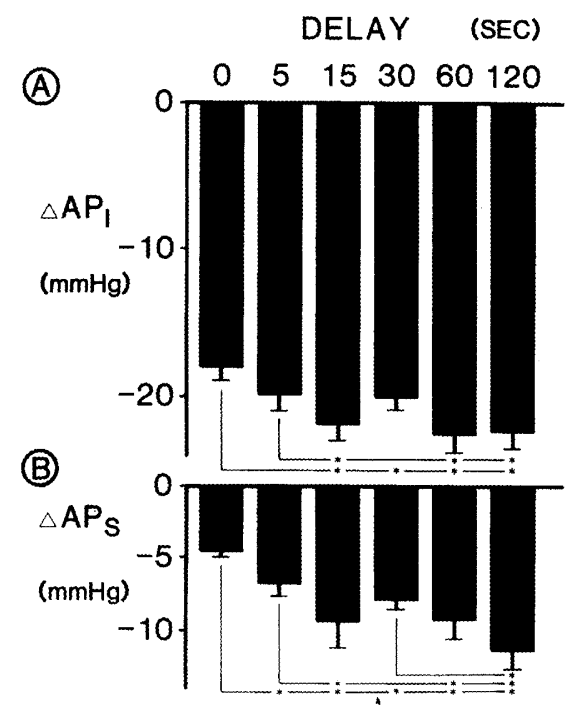

Fig. 3. Immediate $\left(\triangle A P_{1}\right)$ (panel $\left.A\right)$ and steady-state $\left(\triangle A P_{S}\right)$ (panel $\left.B\right)$ falls in mean systemic arterial pressure in response to quick mild hemorrhage preceded by a decrease in intracarotid sinus pressure with different delays (DELAY). The bar and vertical line represent the mean and S.E. $(n=8) .{ }^{*} p<0.1$.

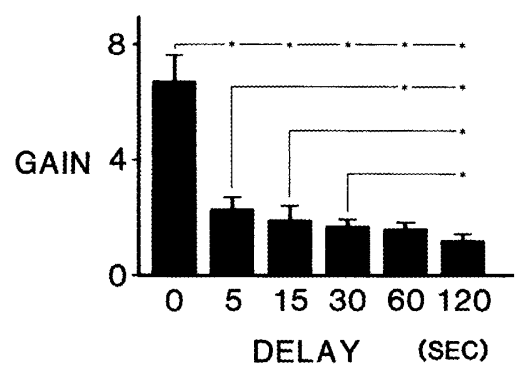

Fig. 4. Effect of preceding activation of the carotid sinus baroreflex system on the open-loop gain of the rapidly acting arterial pressure control system. The gain was estimated from the mean systemic arterial pressure response to quick mild hemorrhage preceded by a decrease in intracarotid sinus pressure with different delays (DELAY). The bar and vertical line represent the mean and S.E. $(n=8)$. ${ }^{*} p<0.1$.

baroreflex system. The after-effect of the preceding activation of the carotid sinus baroreflex system, however, remained significantly in the open-loop gain of the vagally mediated baroreflex system, when the delay was less than $30 \mathrm{~s}(p<0.1)$. 


\section{DISCUSSION}

The present results allow two conclusions. First, the carotid sinus and vagally mediated baroreflex systems summate in a facilitatory manner in compensating for mild posthemorrhage hypotension, when they are activated simultaneously. Second, there is a temporal interaction between the carotid sinus and vagally mediated baroreflex systems, when activation of the vagally mediated baroreflex system is deferred for $30 \mathrm{~s}$ or less after activation of the carotid sinus baroreflex system.

The open-loop gain of the vagally mediated baroreflex system was estimated to be 1.4-1.7 by the quick mild hemorrhage method in the present study. The validity of the quick mild hemorrhage method to estimate the gain of the vagally mediated baroreflex system has been described by KaIzuKa et al. (1985). Employing the same method, the gain has been reported previously to be 1.5 (KAIZUKA et al., 1985) and 1.8 (Hosomi $e t$ al., 1981). The gain value estimated in this paper was very close to these earlier values. These findings imply that the surgical insult in the present study would not affect the pressure-restoring function of the vagally mediated baroreflex system.

The open-loop gain of the vagally mediated baroreflex system, which involves the aortic and cardiopulmonary baroreflex systems, was estimated at different levels of intracarotid sinus pressure in the present experiments. The gain value was not affected by the level of intracarotid sinus pressure. BRUNNER et al. (1984) have reported similar results. They estimated the open-loop gains of the aortic baroreflex system with different levels of intracarotid sinus pressure over the range from 50 to $225 \mathrm{mmHg}$. They found that the level of intracarotid sinus pressure did not affect the gain. They estimated the peak gain to be 0.235 . This gain value does not include the gains of the baroreceptors in the region of the brachiocephalic or subclavian arteries and the cardiopulmonary region. Moreover, this result pertains only to the reflex gain of the systemic arterial resistance, and does not include the reflex gain of the cardiac pumping or vascular capacity. Therefore, this gain value is not comparable to the gain estimated in the present study.

The open-loop gain of the carotid sinus baroreflex system was estimated to be $1.9 \pm 0.3$, when the intracarotid sinus pressure was changed from 140 to $120 \mathrm{mmHg}$. SCHMIDT et al. (1971) changed the intracarotid sinus pressure in steps of $25 \mathrm{mmHg}$ from 50 to $200 \mathrm{mmHg}$ and reported maximum gain (about 1.9) between 100 and $150 \mathrm{mmHg}$. Their experimental conditions and results were comparable to those in our study.

The open-loop gain of the rapidly acting arterial pressure control system was 6.7 , when the carotid sinus and vagally mediated baroreflex systems were activated simultaneously. This value was larger than the sum of the gains of the carotid sinus (1.9) and vagally mediated baroreflex system (1.5). Thus, the carotid sinus and vagally mediated baroreflex systems interact in a facilitatory manner, i.e., spatial interaction. Similar results have been reported by Hosomi and SAGAwA (1979) and 
Hosomi et al. (1981). In these experiments, both the input and output are the arterial pressure changes after the hemorrhage. Therefore, the reflex system which responds to arterial pressure changes after hemorrhage and restores arterial pressure change should be the pressure control system. This was confirmed as follows. After the carotid sinus and vagus nerves were sectioned, the arterial pressure fall caused by hemorrhage was not restored at all within 2 min (Hosomi and SAGAwA, 1979; Hosomi et al., 1981; Hosomi and YoкоYамA, 1981). These results imply that the decomposed reflex systems should be the rapidly acting pressure control systems. KENDRICK et al. (1979) decomposed the rapidly acting arterial pressure control system employing electrical stimulation of the aortic and carotid sinus nerves. They compared the falls in arterial pressure caused by separate and combined electrical stimulations of the aortic and carotid sinus nerve in the dog; simultaneous stimulation caused a fall which was $70 \%$ greater than the sum of the individual depressions. IsHIKAWA and SAGAWA (1983) compared the combined effect of stimulation of a unilateral aortic nerve and a simultaneous increase in bilateral carotid sinus pressures from 40 to $70-80 \mathrm{mmHg}$, with the sum of these effects measured separately. The combined effect was always larger than the sum of the separate effects.

There is much disparity in the literature regarding the interaction of carotid and aortic reflexes or carotid and vagally mediated baroreflexes. This problem has recently been the subject of a thorough review by SAGAwa (1983). Perhaps such conflicting results could be explained, if the manner of studying the interaction was clearly defined. ANGELl-JAMES and DALY (1970) investigated the summation by comparing the changes in total systemic resistance during combined and separated increases in isolated carotid sinuses and aortic arch pressure. Their data indicated a mildly inhibitory summation. Severe surgical insult could be a possible cause of this finding. Moreover, the decomposed system was the baroreflex control system of the total systemic resistance. NiNOMIYA and IRISAWA (1969) found an inhibitory summation for the sino-aortic inhibition of renal nerve discharge. The system studied was the baroreflex control system of renal nerve activity. Ноsomi et al. (1986) analyzed the summation of the two reflexes activated by quick mild hemorrhage on the perfusion pressure of a vascularly isolated hindlimb, and found a linear summation (or simple addition). In this experiment, the decomposed system was the baroreflex control system of the hindlimb perfusion pressure. Thus, the difference in input or output used to decompose the reflex system from the whole organism could result in different interactions. The system studied here was the baroreflex system, i.e., the rapidly acting arterial pressure control system, and may differ from the systems described above. IshiKaWA and SAGAwA (1983) noted another reason for disparity. They used isolated carotid sinus perfusion and aortic nerve stimulation in the rabbit to demonstrate that interactions of the carotid and aortic reflexes could be described as facilitatory or inhibitory depending on the input level. The operating range of the baroreceptors has to be in the physiological range in studying interactions. In the present study (Protocol 3), the baroreceptors 
were unloaded from the physiological range of $100-140 \mathrm{mmHg}$.

Another important finding was the existence of a temporal interaction between the carotid sinus and vagally mediated baroreflex systems, if the latter was activated by hypotension with a delay of less than $30 \mathrm{~s}$ after activation of the carotid sinus baroreflex system. The definition of temporal interaction is that when two stimuli are applied in close succession, the response to the second stimulus is affected by the preceding one. BRUNNER et al. (1984) have employed the term "temporal interaction" differently. They defined "temporal interaction" as the interaction of reflex systems when stimuli are delivered to both receptors at the same time. Therefore, the term "temporal interaction" as used by BRUNNER et al. (1984) corresponds to the "spatial interaction" in this paper.

KATONA and TAN (1975) changed the relative timing of aortic and carotid sinus baroreceptor activity by electric stimulation of the nerves. They altered the relative delay from 0 to 0.75 of the average time duration of the cardiac cycle and found that changing the relative delay between aortic and carotid sinus nerve stimulation did not result in blood pressure variations in the rabbit. KENDRICK et al. (1979) studied the cardiovascular response to combined simultaneous stimulation of the aortic nerve and carotid sinus nerve with trains of electrical stimuli in dogs. They found a strong facilitatory summation. The degree of interaction between the reflexes did not change as the delay between the stimulus trains was varied over an interval of 0 and $100 \mathrm{~ms}$. We have no definite explanation for this discrepancy. In these two studies, electrical stimulation rather than pressure stimulation of the aortic arch and carotid sinus baroreceptor reflexes was used. However, the techniques employed in these two groups are open to the criticism that abnormal discharge patterns are produced in the nerves, chemosensory as well as barosensory fibers may be excited, and the ratio of large to small barosensory fibers activated may be abnormal. We can reasonably assume that the stimulation parameters may not be precisely equal to each other, and consequently different responses may be obtained.

KÖTTER et al. (1970) found that the pressure reduction caused by stimulation of the carotid sinus nerve at a fixed frequency is enhanced if preceded by a $30 \mathrm{~s}$ stimulation of the aortic nerve at a higher frequency. This observation seems to be similar to our findings. In their work all stimuli, however, were electrical and of supramaximal intensity, and so the observed effects cannot be attributed to baroreceptors alone. Moreover, the stimulation sequence of the baroreceptors in their study was entirely different from that in ours. The reflexogenic areas examined in their experiments were the aortic arch and carotid sinus baroreceptor regions, but those in our study were the carotid sinus baroreceptor region and the vagally mediated baroreceptor regions, including the aortic arch and cardiopulmonary baroreceptor regions. Therefore, our data cannot be compared with theirs.

A possible explanation of the temporal interaction is that a resetting of the baroreceptors of the vagally mediated baroreflex system can increase $G_{\mathbf{V}}$. A decrease in the intracarotid sinus pressure causes a sustained increase in the systemic arterial pressure. The increase in the systemic arterial pressure can cause a right shift 
(resetting) of operating range of the receptors of the vagally mediated baroreflex system (UNDESSER et al., 1984). The resetting in itself does not change an open-loop gain of the system. The right shift of an operating range of the aortic arch baroreflex system, however, may cause an increase in the open-loop gain. Thus, the resetting may increase the gain of the vagally mediated baroreflex system.

Our results suggest that the spatial interaction between the carotid sinus and vagally mediated baroreflex systems is facilitatory. A temporal interaction between both systems is obvious, if the vagally mediated baroreflex system is activated within $30 \mathrm{~s}$ after activation of the carotid sinus baroreflex system.

This work was supported in part by a grant from Professor Isamu Nishida and a Grantin-Aid for Scientific Research (No. 59570039) from the Ministry of Education, Science and Culture of Japan.

\section{REFERENCES}

ANGell-JAmes, J. E. and Daly, M. DE B. (1970) Comparison of the reflex vasomotor responses to separate and combined stimulation of the carotid sinus and aortic arch baroreceptors by pulsatile and non-pulsatile pressures in the dog. J. Physiol. (Lond.), 209: 257-293.

Brunner, M. J., Greene, A. S., Kallman, C. H., and Shoukas, A. A. (1984) Interaction of canine carotid sinus and aortic arch baroreflexes in the control of total peripheral resistance. Circ. Res., 55: 740-750.

Hosomi, H., Baba, M., and Morita, H. (1981) Time-dependent changes in open-loop gains of baroreflex systems after massive hemorrhage. Jpn. J. Physiol., 31: 705-715.

Hosomi, H., Chatani, H., Kaizuka, T., Katsuda, S., and Hayashida, Y. (1983) Overall open-loop gain of rapidly acting arterial pressure control system in rabbits. Pflügers Arch., 399: 134-138.

Hosomi, H., Kawata, M., and Koyama, S. (1986) Effects of phenylephrine on the responsiveness of baroreceptor reflex systems, In: Brain and Blood Pressure Control, ed. by NaKAMURA, K., Elsevier, Amsterdam, pp. 171-176.

Hosomi, H. and SAGAWA, K. (1979) Sinovagal interaction in arterial pressure restoration after 10\% hemorrhage. Am. J. Physiol., 237: R203-R209.

Hosomi, H. and Yокоуама, K. (1981) Estimation of open-loop gain of canine arterial pressure control system by a new method. Am. J. Physiol., 240: H832-H836.

IsHIKAWA, N. and SAGAWA, K. (1983) Nonlinear summation of depressor effects of carotid sinus pressure changes and aortic nerve stimulation in the rabbit. Circ. Res., 52: 401410.

Kaizuka, T., Yoshida, H., and Hosomi, H. (1985) Time-dependent effect of sectioning carotid sinus nerves on the vagally mediated baroreflex. Pflügers Arch., 403: 258-261.

KatONA, P. G. and TAN, K. S. (1975) Interaction of aortic and carotid sinus baroreceptors: Effect of activation times. Am. J. Physiol., 228: 238-243.

Kendrick, J. E., Matson, G. L., and Lalley, P. M. (1979) Central interaction between the baroreceptor reflexes from the carotid sinus and aortic arch. Am. J. Physiol, 236: H127-H133.

Kötter, V., Zerbst, E., and Schulze, A. (1970) Untersuchungen zur Bahnung und 
Konvergenz pressoreceptorischer Afferenzen mit Hilfe der KonditionsTestreiztechnik. Pflügers Arch., 320: 210-232.

MilhoRn, H. T., Jr. (1966) The Application of Control Theory to Physiological Systems, Saunders, London, pp. 156-166.

NinOMIYA, I. and IRISAWA, H. (1969) Summation of baroreceptor reflex effects on sympathetic nerve activities. Am. J. Physiol., 216: 1330-1336.

SAGAWA, K. (1983) Baroreflex control of systemic arterial pressure and vascular bed. In: The Handbook of Physiology, ed. by ShePherd, J. T. and ABboud, F. M., American Physiological Society, Washington, D.C., pp. 453-496.

Schmidt, R. M., Kumada, M., and SAGaWA, K. (1971) Cardiac output and total peripheral resistance in carotid sinus reflex. Am. J. Physiol., 221: 480-487.

UNDESSER, K. P., LYNN, M. P., and BishoP, V. S. (1984) Rapid resetting of aortic nerves in conscious rabbits. Am. J. Physiol., 246: H302-H305. 